\title{
fAPEbook - Animal Social Life Monitoring with Wireless Sensor and Actor Networks
}

\author{
Mustafa İlhan Akbaş*, Matthias R. Brust*, Carlos H.C. Ribeiro ${ }^{\dagger}$, and Damla Turgut* \\ ${ }^{*}$ Department of Electrical Engineering and Computer Science \\ University of Central Florida, Orlando FL \\ Email: \{miakbas,mbrust,turgut $\} @$ eecs.ucf.edu \\ ${ }^{\dagger}$ Computer Science Division \\ Technological Institute of Aeronautics, Brazil \\ Email: carlos@ita.br
}

\begin{abstract}
Wild life monitoring requires a sophisticated process of planning, installation, execution, data collection, and data interpretation. The effort and time spent increases tremendously with the area and the number of observed objects as well as with the time frame of the observations. However, the application of wireless sensor nodes enables a scalable sampling method and fine granularity of data difficult to obtain otherwise. By including resource rich actor nodes, the data collection and evaluation are further optimized.

In this paper, a wireless sensor and actor network (WSAN) protocol is designed for capturing and monitoring the social interactions of the complex social network of gorillas. The nodes are intended to be attached on the apes forming a mobile network. The local interaction patterns among the nodes are analyzed throughout the network life time. The protocol then determines the social roles of the gorillas based on previous research findings about their social structure, and builds a profile for each ape, which demonstrates the status, kinship and role played within the society. As a result, the proposed protocol generates a social directory of the ape troop under observation, namely fAPEbook. The contribution of this paper is to evaluate the efficiency of the proposed algorithm by its ability to capture the different characteristics in the society with the established mobility models. The results suggest the applicability of the algorithm in field tests with wild life.
\end{abstract}

\section{INTRODUCTION}

Monitoring the environment or the behavior and habitat of animals is a challenging task, since it involves a sophisticated process of planning, installation, execution, data collection, and data interpretation. Due to the complexity, the traditional approaches for wild life monitoring have to deal with a number of challenges [1]: (a) most methods are highly invasive on the environment and objects under study, in particular, when periodical resampling is needed, (b) the choice of the right sampling method that delivers the requested data is crucial, (c) the effort to plan and execute a monitoring project tremendously increases with the area size and number of objects under investigation as well as the period of observation.

Monitoring requires an appropriate design of the sampling process to guarantee accurate and precise measurements, which are particularly challenging when monitoring an animal population changing its habitat frequently. The latest advances in wireless networks can be used to overcome many of these drawbacks and challenges.

Wireless sensor and actor networks (WSANs) [2] consist of tiny sensor nodes sensing the events and a limited number of actors, which can actively collect, process the information and react to the events. A sensor node has limited data processing capability, transmission rate, energy, and memory. Actors, on the other hand, possess increased computation capabilities, wider communication ranges, long lasting batteries and larger memories. Improvements in capabilities of sensors as well as the self-organizing aspect of the participating nodes make WSANs a state-of-the-art tool for wild life monitoring and in particular for wild social life.

In this paper we describe algorithms for data collection within a WSAN and specially designed algorithms for data evaluation. More specifically, we consider the capturing, monitoring and evaluation of gorilla social life. Gorillas build and live in a hierarchical society and a group of gorillas that belong to the same social network is called a "troop". Many properties of gorilla social life are already known [3], but since the lifespan of gorillas can vary between 30-50 years with gorillas reproducing and leaving the troop and with the troop constantly moving to new habitats, many more aspects of their social life remain unknown. The gorilla troop has a strict social organization, and it remains a challenge for scientists to find out how gorillas interact and how their affiliations might change without interrupting the troop due to the presence of cameras or observers.

By equipping the gorillas with sensors, the resulting WSAN can capture essential characteristics of each gorilla such as its interactions with its environment or troop members, its movement, speed and so on.

fAPEbook uses the collection of the spatial-temporal relationships to build up a profile for each gorilla in the distributed system of WSANs, which can be seen as the Facebook [4] for that Gorilla troop. However it must be stressed that each profile is generated automatically and locally. 


\section{RELATED WORK}

There are various studies on the deployment of sensor nodes for wild life animal tracking. Environmental scientists and zoologists have been increasingly using these technologies to collect data from wild terrestrial areas and transmit them to the remote databases [5]. In some of these applications, the sensor nodes are attached to the animals, forming an ad hoc wireless network of mobile nodes [6].

ZebraNet [7] is one of the initial examples of such studies, in which the animals carry custom tracking collars with GPS capability and form a mobile sensor network across a large area. The collars include sensor nodes with the global positioning system (GPS) capability, and they form the WSN to monitor the area and record the data. Wark et al. [6] applies sensor network technology to farming and their approach also includes collars worn by animals. This system utilizes both static and mobile nodes measuring the state of a complex, dynamic system comprising climate, soil, pasture, and animals. Naumowicz et al. [8] deployed a WSN on Skomer Island, Wales to improve the investigation of the behavior and spatial ecology of the Manx Shearwater seabirds. The system informs the scientists with high resolution data about the arrival and departure of the birds and the environmental parameters such as temperature or humidity. Handcock et al. [9] uses a large cattle enterprise to demonstrate the potential for combining GPS collars and satellite images in a WSN. The implemented WSN is used to monitor behavioral preferences and social behavior of cattles in Northern Australia. There are also sensor network applications where the sensor nodes are implanted within the animal body in order to monitor the functioning of the whole body or a particular organ of the animal (e.g [10]).

In this paper, several basic concepts about the movement of the gorilla troops and the social hierarchy of the troops, which can be found in the research literature on gorilla behavior, are used. For instance, Fossey [11] conducted the initial studies on home ranges of the mountain gorillas and their social interactions. Fossey's observations include hand drawn maps of mountain gorilla ranging paths that form the basis for movement patterns of the gorilla troops. The gorilla behaviors and social interactions in troops have been analyzed in various studies [3], [12].

\section{FAPEBOOK}

\section{A. Ape social structure}

Gorillas are socially organized and live in troops. A troop is usually comprised of 3 to 30 individuals. According to gender, age, strength and affinity, the individual animals in the troop have different roles:

- Silverback: A silverback is the dominant male leading the group in their daily travels. The silverback has exclusive breeding rights to the females. Generally there is one leader silverback in each troop.

- Adult female: Different than other primates, adult females do not interact with each other and usually compete to stay close to the silverback. Generally there are three or four adult females in each troop.

- Blackback: Juvenile males tend to stay not very close to silverbacks and may attempt to leave the troop to form a breeding group.

- Juvenile female: A juvenile female stays closer to the troop compared to blackbacks and may change family groups a number of times.

- Newborn and Infant: A newborn forms a very close relationship to its mother, rarely straying more than a few steps from her side for three to four years.

Maturing males generally leave their family groups and these wandering males follow other bands to entice females to join them. The example structure of a gorilla society is shown in Fig. 1 . Gorillas have complex social lives with families, affections, and politics of their own. The information used in our approach follow the general guidelines, considering well known facts about gorillas into account. For instance they are not fast moving animals, they are not territorial and they move about 400 to 1000 meters everyday.
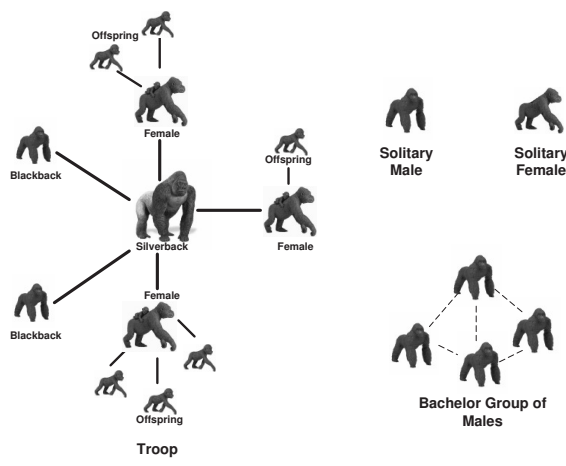

Figure 1. Gorilla social structure.

\section{B. System model}

We assume that each gorilla is equipped with a wireless sensor node except the silverbacks, which are equipped with the actors. On some rare points in the habitat, gateway actor nodes are installed as shown in Figure 2 to collect data from the actor network when they are in transmission range and to form the backbone of the network.

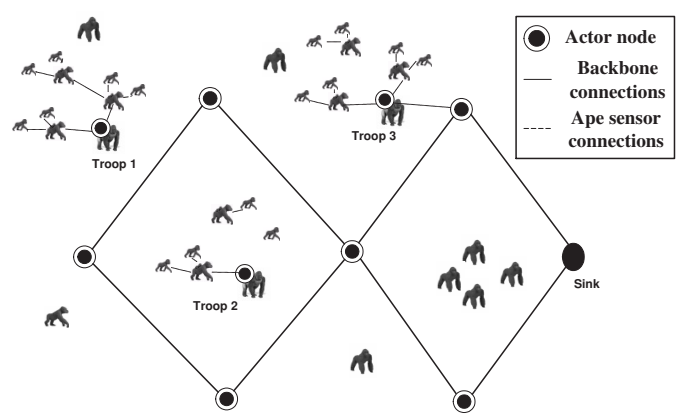

Figure 2. Application scenario of three troops and some single gorillas. 
All communication and packet exchange in the network occurs only locally. When communicating with the sensor nodes and creating the affiliation areas, the actors use the same transmission range, $r_{s}$, as the sensor nodes exchange packets only with their neighboring sensor nodes. Each actor node encodes the packets transmitted with its ID and weight $k$ to update the weight of the sensor nodes.

\section{Mobility pattern}

Lévy walk is an optimal way to find randomly dispersed objects [13]. It is observed as the mobility model in most of the animal foraging patterns, such as jackals [14] or spider monkeys [15]. Lévy walk is a random walk with step-lengths distributed according to a heavy-tailed probability distribution. The distribution is a power law of the form $y=x^{-\alpha}$ where $1<\alpha<3$. Each step in Lévy walk is a tuple $L=(1, \theta, \Delta t f, \Delta t p) . \Delta t f$ indicates the flight duration and it is chosen for each walk from a probability distribution $p(l)$. $\Delta t p$ specifies pause time at the end of a walk and $\theta$ is the random direction taken by a node.

Immitating gorilla groups in the nature, the main path of the troop is determined by the movement of the silverback, for which the Levy walk mobility is used. After the determination of the main path of the troop, the center of mass (CoM) concept of physics is used as an addition to Levy walk to determine the positions of all animals in the swarm. The CoM approach is used to include the natural clustered structure of gorilla troops. For instance, the breeding females form a group and their CoM is taken as the silverback. The coordinates of the nodes around the silverback must satisfy $\vec{p}_{s}=\sum_{i=1}^{N} \vec{p}_{i}$, where $N$ is the number of nodes around the silverback and $\vec{p}_{s}$ is the position of the silverback. This method is applicable and modifiable for any animal swarm.

\section{Data collection}

The main goal of the system is to monitor the social behavior of gorillas, therefore the social structure is utilized in the design of our protocol. Since the silverbacks are the leaders of their troop and the other members tend to stay close, the silverback becomes the natural clusterhead of his troop. Additionally, there are actors in the network attached to the silverbacks, which are mobile due to the movements of the silverbacks. Consequently, the network is composed of mobile nodes (sensors and actors) and a backbone of stationary actors. This architecture is suitable to use a clustering approach for the network organization part since the actors match very well to the predetermined clusterhead roles.

The network structure is formed and maintained by the state-transition rules of Algorithm 1. The algorithm uses local information and all nodes rapidly update their data as the network structure changes. A sensor node keeps only the maximum weight for each actor it receives packets from and it does not build a state or history of the whole network. When a sensor node receives a packet from an actor, the packet is retransmitted if its weight is less than the sensor node's weight for that actor. Otherwise the sensor node drops the packet to avoid unnecessary traffic and energy consumption in the network. The weight $w$ of each affiliation depends on the $k$ hop distance of a node to an actor. The condition, in which a sensor node does not receive any weight updates, is defined as the "loss of connection" for the sensor node. This may refer to a solitary gorilla in the area. In that case, the sensor node sets its hop value to the minimum value defined for the network. Then it operates only in "listening" mode and does not transmit any packets.

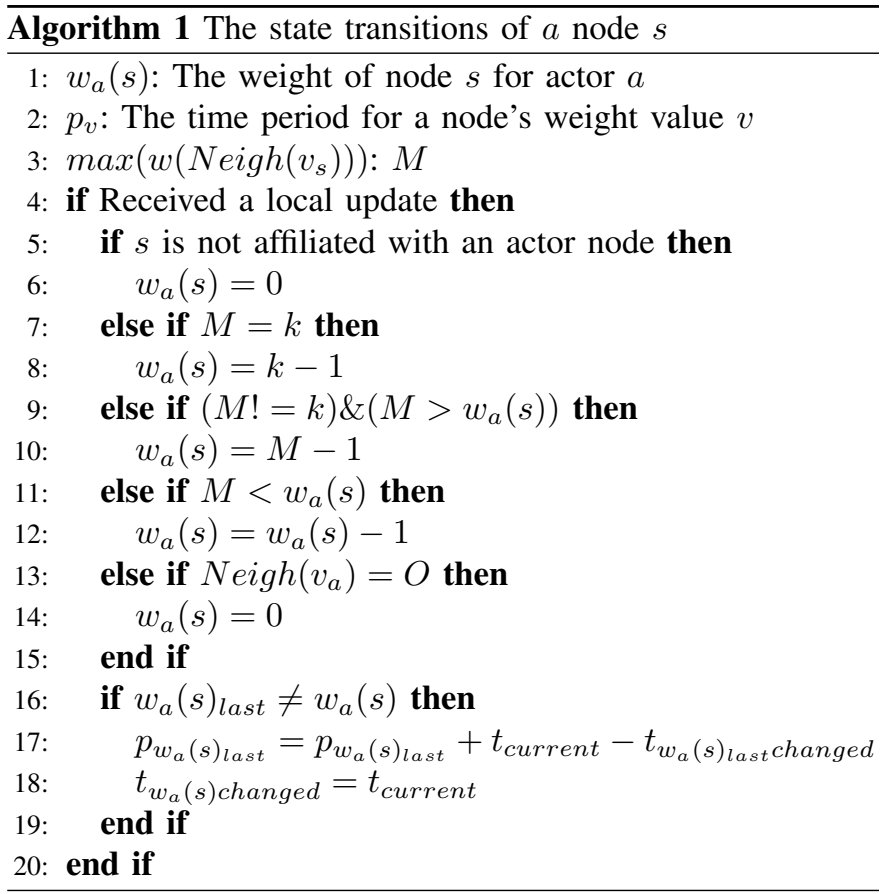

Algorithm 1 utilizes the spatial proximity to decide on the weights of the sensor nodes. The weight information for the nodes in a troop is collected at the corresponding mobile actor and transferred to the backbone whenever it is possible. The periods of time for specific weight values are also calculated in Algorithm 1 to be employed by actors. Information about a troop can be collected by more than one actor. All of the collected data are integrated at the sink to decide on the social structure of the gorilla population.

Algorithm 2 interprets the role of each gorilla by using the information gathered from the network and the rules designed for the animal society, which is the subject of the study. The transmission range, time and cardinality are the parameters defining and quantifying the rules. The thresholds of these values can be generalized according to the troop under observation while the rules are held very specific for the social network of gorillas.

fAPEbook uses feedback from the system to take new decisions as shown in Fig. 3. As the mobile actors collect information from the network and bring it to the backbone, the sink will update the roles of the gorillas depending on the feedback. If an ape is determined as a female with offspring, the distance analysis is executed with varying transmission ranges to find the distances of the offspring. This is used 


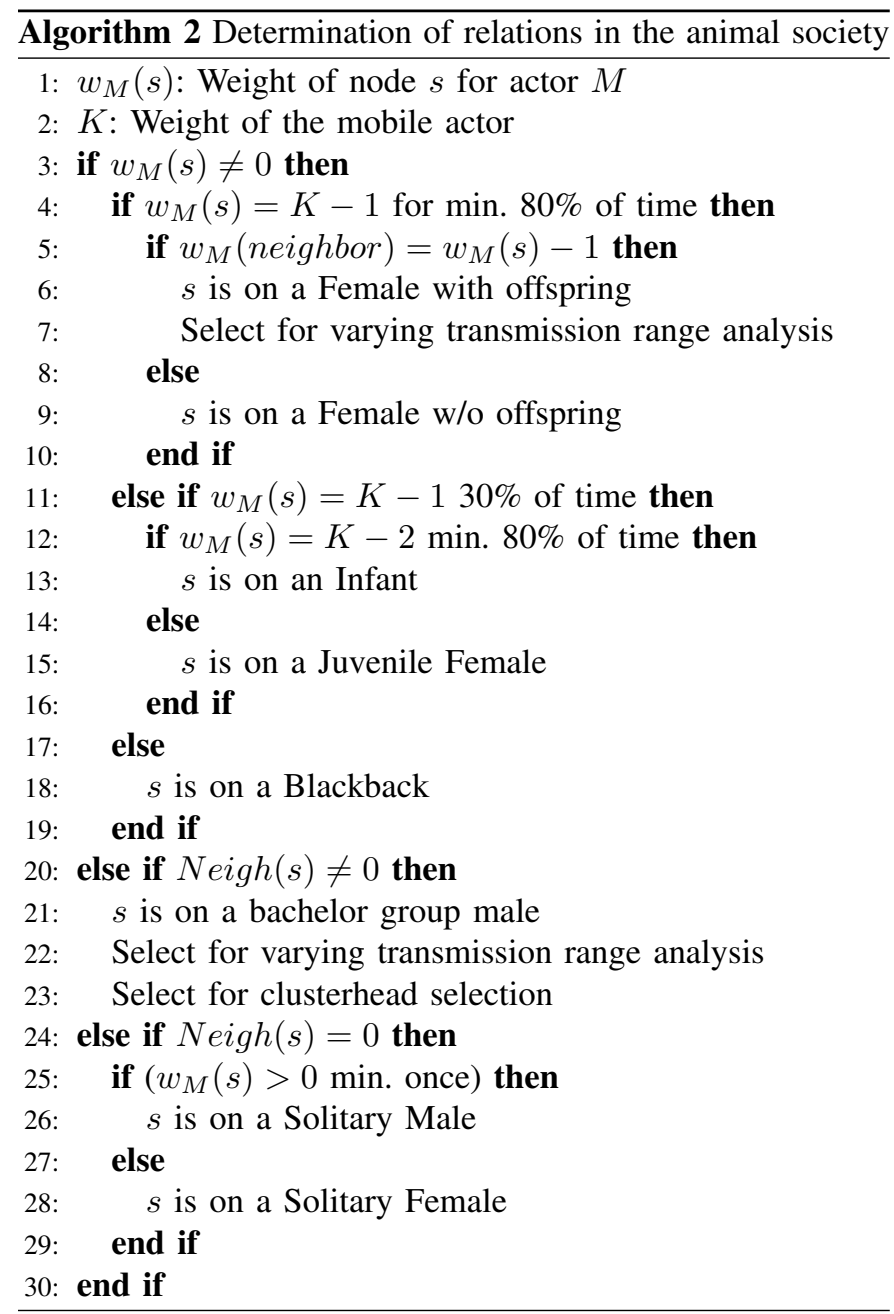

as a method to decide on the ages of the infants since they stay close to the mother ape for most of the day as well as when they are sleeping. Additionally, this analysis helps to decide on the data aggregation points in the network since mother females form perfect data aggregation points for the data gathered from the offspring. If the feedback from the network shows that a group of blackbacks form a bachelor group, one of these blackbacks is chosen as the data collection gateway for the group. This is efficient both in terms of data collection and energy efficiency.

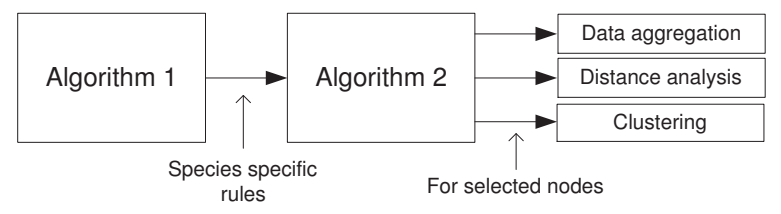

Figure 3. Algorithm chart.

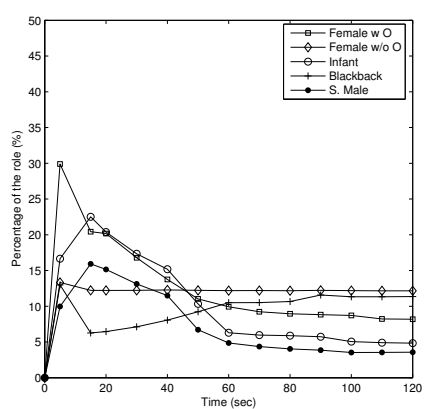

(a) Members of the troop.

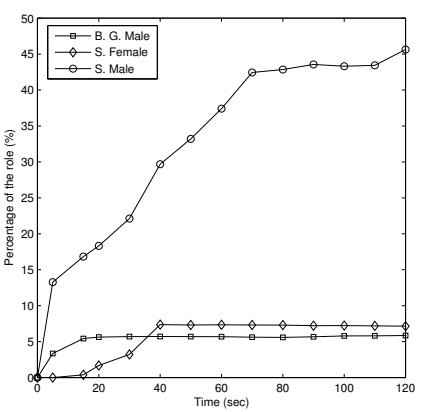

(b) Non-members of the troop.
Figure 4. Percentages of the roles vs. time in random walk.

\section{Simulation Study}

\section{A. Simulation environment and metrics}

The simulations are conducted in the OPNET modeler [16]. IEEE 802.11 is the underlying MAC layer for sensor and actor nodes. The transmission range of a sensor node is taken as 10 meters. In order to simulate the nodes in the network, 1 actor and 30 sensor nodes are positioned in the simulation area of $1750 \times 1750$ meters. Lévy walk mobility model is used with $\alpha=1.9$ and $\beta=1.6$, which are taken according to observed values of the animal foraging patterns [15]. Random waypoint mobility model is used with speed uniformly distributed in $(3,8) \mathrm{km} / \mathrm{h}$ interval and pause time uniformly distributed in $(50,150)$ seconds interval. The values of these metrics are determined according to the standard literature on gorillas [11].

\section{B. Simulation Results}

1) Roles: In the first set of experiments, the nodes move according to the Random waypoint mobility pattern. Twenty simulation runs were executed with the same initial conditions and the average percentage of roles are determined by fAPEbook. In Fig. 4 (a), the percentages of the troop members over the simulation period are given. The percentages of the animals, which are not members of the troop, are given in Fig. 4 (b).

Fig. 4 shows that there is an increase in the number of solitary males with the simulation time. The other roles have shares similar to each other, mostly depending on their initial positions and their directions. The solitary males in the nature walk alone in the habitat and they generally get affiliated with troops over time, but can not become a member quickly. Hence this is an expected property for the society with random mobility with a starting condition in which most of the nodes are close to each other. Most of the nodes get departed from the troop as the time passes and fAPEbook marks them as solitary males when they range alone in the area.

In the next set of experiments, the nodes move with Lévy walk with center of mass mobility pattern. Twenty simulation runs were executed with the same initial conditions as in the initial set of experiments and the average percentage of roles are determined by fAPEbook. The change in the percentage of the members and the non-members of the troop over simulation time are given in Fig. 5. 


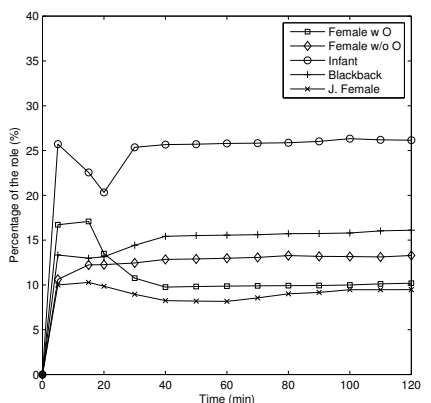

(a) Members of the troop.

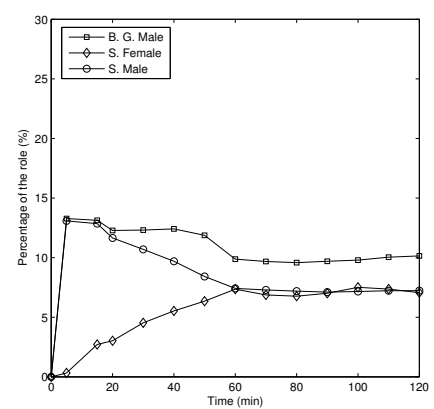

(b) Non-members of the troop.
Figure 5. Roles vs. time in Lévy walk with center of mass approach.

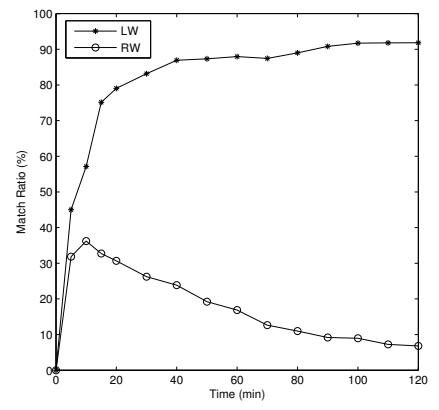

(a) All roles in the society.

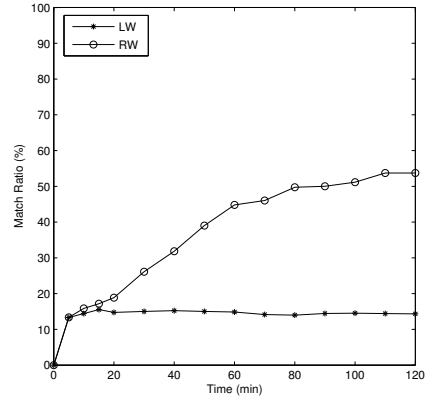

(b) Solitary animals in the society.
Figure 6. Behavior of the troop according to two different metrics.

Fig. 5 shows that the percentage of each role fluctuates at the beginning of the simulation akin to the case of random mobility. This characteristic can be considered as the warm-up period of fAPEbook and it is the first important result of this experiment set. After the warm-up period, the roles in Lévy walk become stable and the roles match about $91 \%$ with the starting roles. The second important result of the experiment shows that Lévy walk with center of mass approach is an appropriate mobility model for fAPEbook simulations, since the used rules match the structure of the real gorilla society network.

2) Social features: While fAPEbook is initially designed to be implemented for a gorilla society, it can be applied to any other animal swarm by modifying the rules in Algorithm 2 or by creating new metrics. For instance, the graphs in Fig. 6 show results for two different metrics chosen for the simulation scenarios. For the first case, we assume that the role distribution of the mobile society must be the same as the role distribution in the stationary case. The metric for Fig. 6 (a) is the ratio of roles distributed in the mobile scenario to the roles distributed in the stationary case. For Fig. 6 (b), the used metric is the ratio of solitary animals in the whole society.

\section{CONCLUSION}

In this paper, a WSAN protocol is proposed to monitor and capture the social network of an ape society. In case of gorillas, it is shown how the data about the gorilla social structure in general aids in the design of an efficient protocol for capturing the social network of a specific gorilla troop. Simulation results show that the fluctuations in the expected match ratio can help discovering new behaviors and roles in the specific gorilla troops. The goal of this study is to capture the social aspects of gorillas, i.e. behavior, affilation, affinity and build a social directory similar to Facebook. In strong contrast to Facebook, however, the protocol generates the profiles of gorillas by using the observed spatial-temporal interaction patterns. This paper presents the study inital to this social directory and the next steps include conducting the field tests.

\section{ACKNOWLEDGEMENT}

The authors would like to thank OPNET Technologies Inc. for supporting this research by providing OPNET Modeler software under OPNET University Program.

Matthias R. Brust gratefully acknowledges support by FAPESP (Fundação de Amparo á Pesquisa do Estado de São Paulo) under grant AP.PIPE 1 2010/51435-9 and RedeAlumni.

\section{REFERENCES}

[1] M. L. Morrison, Wildlife restoration: techniques for habitat analysis and animal monitoring. Island Press, 2002.

[2] R. Verdone, D. Dardari, G. Mazzini, and A. Conti, Wireless Sensor and Actuator Networks: Technologies, Analysis and Design. Academic Press, 2008.

[3] M. M. Robbins and A. McNeilage, "Home range and frugivory patterns of mountain gorillas in bwindi impenetrable national park, uganda," International Journal of Primatology, vol. 24, no. 3, pp. 467-491, 2003.

[4] "facebook," http://www.facebook.com.

[5] J. K. Hart and K. Martinez, "Environmental sensor networks: A revolution in the earth system science?" Earth-Science Reviews, vol. 78, no. 3-4, pp. 177 - 191, 2006.

[6] T. Wark, P. Corke, P. Sikka, L. Klingbeil, Y. Guo, C. Crossman, P. Valencia, D. Swain, and G. Bishop-Hurley, "Transforming agriculture through pervasive wireless sensor networks," IEEE Pervasive Computing, vol. 6 , pp. 50-57, April 2007.

[7] P. Juang, H. Oki, Y. Wang, M. Martonosi, L. S. Peh, and D. Rubenstein, "Energy-efficient computing for wildlife tracking: design tradeoffs and early experiences with zebranet," SIGPLAN Not., vol. 37, pp. 96-107, October 2002

[8] T. Naumowicz, R. Freeman, A. Heil, M. Calsyn, E. Hellmich, A. Brändle, T. Guilford, and J. Schiller, "Autonomous monitoring of vulnerable habitats using a wireless sensor network," in Proc. of the REALWSN, March 2008, pp. 51-55.

[9] R. N. Handcock, D. L. Swain, G. J. Bishop-Hurley, K. P. Patison, T. Wark, P. Valencia, P. Corke, and C. J. O'Neill, "Monitoring animal behaviour and environmental interactions using wireless sensor networks, gps collars and satellite remote sensing," Sensors, vol. 9, no. 5, pp. 3586-3603, 2009.

[10] N. Das, P. Ghosh, and A. Sen, "Approximation algorithm for avoiding hotspot formation of sensor networks for temperature sensitive environments," in Proc. of the IEEE GLOBECOM, 2009, pp. 4566-4571.

[11] D. Fossey, "Observations on the home range of one group of mountain gorilla (gorilla gorilla beringei)," Animal Behaviour, vol. 22, pp. 568 581,1974

[12] M. Boos, M. Kolbe, P. Kappeler, and E. T., Coordination in Human and Primate Groups. Springer-Verlag New York, Inc., 2011.

[13] G. M. Viswanathan, S. V. Buldyrev, S. Havlin, M. da Luz, E. P. Raposo, and H. E. Stanley, "Optimizing the success of random searches," Nature, vol. 401, pp. 911-914, 1999.

[14] R. P. D. Atkinson, C. J. Rhodes, D. W. Macdonald, and R. M. Anderson, "Scale-free dynamics in the movement patterns of jackals," OIKOS, vol. 98, pp. 134-140, 2002.

[15] J. Ramos-Fernandez, L. Morales, O. Miramontes, G. Cocho, H. Larralde, and B. Ayala-Orozco, "Levy walk patterns in the foraging movements of spider monkeys (ateles geoffroyi)," Behavioural Ecology and Sociobiology, vol. 55, pp. 223-230, 2004.

[16] "Opnet modeler," http://www.opnet.com. 